\title{
TRANSPOSED TRANSLATION PLANES
}

\author{
D. M. MADURAM
}

ABSTRACT. A detailed study of the transposed translation planes which are also given by dual spreads is made using the matrix representation of translation planes.

I. Introduction. Knuth [8] gives a method of constructing a new semifield plane from any given semifield plane by transposing its corresponding cubical array [6, p. 239], and he asserts the possibility of extending this construction to arbitrary quasifields $[8, \$ 5.1]$. Using the matrix representation of translation planes [2, 55 , we give a proof of the assertion and establish the equivalence of transposed translation plane to the translation plane over the corresponding dual spread [4]. Bruen [4] discusses the notion of a dual spread and the relation between the collineation group of the translation plane defined, respectively, by a spread and its dual. What we do amounts to showing that the notions of a dual spread and of the transposed plane are equivalent. Some of what we do is implicit in Bruen; the correspondences are given in explicit form here. In particular, we give an explicit mapping showing an isomorphism between the translation complements of a plane and of its transpose. (In vector space representation, the translation complement is the group of collineations fixing the zero vector.)

\section{Known results.}

Definition. A spread $S$ of the vector space $V_{2 n}$ of dimension $2 n$ over the Galois field $F=G F(q)$ is a set of $n$-dimensional subspaces, which are called components such that each nonzero element of $V_{2 n}$ belongs to exactly one component.

Definition. A spread $S^{\prime}$ of the vector space $V_{2 n}$ is dual to a spread $S$ of $V_{2 n}$ if there exists a correlation in $V_{2 n}$, considered as a projective space of dimension $2 n-1$, which maps each component of the spread $S$ onto that of the spread $S^{\circ}$.

Result 1 [11, p. 49]. Any translation plane can be considered as a plane of the form $P\left(V_{2 n}, S, F\right)$ where points are the vectors of $V_{2 n}$ and the

Received by the editors April 1, 1974 and, in revised form, July 30, 1974 and October 15, 1974.

AMS (MOS) subject classifications (1970). Primary 20B25, 50D35; Secondary 20N10, 05B25.

Key words and phrases. Translation planes, transposed translation planes, spreads, dual spreads, quasifields and nucleus. 
lines are the components of the spread $S$ and their translates under the group of translations of $V_{2 n}$.

Using the matrix representation of translation planes, we have the following well-known result.

Result 2 [11, p. 51]. Any translation plane is isomorphic to a plane of the form $P\left(V_{2 n}, S, F\right)$ in which the components of the spread $S$ are given by the equations $x=0, y=0, x=y^{\prime}=x T_{0}, y^{\prime}=x T_{i}$, where $i=1,2, \cdots$, $q^{n}-2$ with $V_{2 n}=V_{n}+V_{n}, V_{n}=F^{n}$ as a vector space and the $n \times n$ matrices $T_{i}$ satisfy the following property: $T_{i} T_{j}^{-1}$ is either fixed point free or identity.

It is easy to see that the converse holds. We can state this precisely with the following

Definition. A set of $n \times n$ matrices $\left\{I=T_{0}, T_{1}, \ldots, T_{N}: N=q^{n}-2\right\}$ over the field $F=G F(q)$ for any prime power $q$ forms a matrix representative set if $T_{i} T_{j}^{-1}$ is either fixed point free or identity.

Result 3. There exists a canonical surjection from matrix representative sets onto translation planes.

Result 1. [3, Lemma 10.1]. If $\left\{T_{i}\right\}$ forms a matrix representative set, then $\left\{T_{i}^{-1}\right\}$ forms again a representative set giving an isomorphic translation plane.

Translation planes can be studied algebraically also. With each transla. tion plane and a choice of coordinate system with $U, V$ on the line at infinity, we can associate a quasifield. A quasifield can be constructed directly from the matrix representative set as follows:

The elements of the quasifield are the elements of $V_{n}$. Addition in the quasifield is the ordinary addition of vectors, and multiplication in the quasifield is given by the rule, $0 \cdot b=b \cdot 0=0$ for $b$ in $V_{n}$ and for a fixed $e$ in $V_{n}, c T_{i} \cdot b=b T_{i}$ for $b$ in $V_{n}$ and $T_{i}$ in the matrix representative set. More precisely, for $a, b \neq 0$ in $V_{n}$,

$$
a \cdot b=b T_{i}
$$

where $T_{i}$ is the matrix of the representative set corresponding to the component containing the point $(e, a)$.

\section{Transposed translation planes.}

Proposition l. If $\left\{T_{i}\right\}$ forms a matrix representative set, then $\left\{T_{i}^{t}\right\}$, where $t$ denotes transposition of a matrix, also forms a representative set.

Proof. Apply Results 3 and 4.

Proposition 2. If a spread $S$ determines a translation plane with representative set $\left\{T_{i}\right\}$, then its dual spread determines the corresponding transposed translation plane with the representative set $\left\{T_{i}^{t}\right\}$. 
Proof. Considering the vector space $V_{2 n}$ as a projective space of dimension $2 n-1$, any component of the spread given by the matrix $T_{i}$ consists of points represented by the vectors $\left(x, x T_{i}\right)$.

Now consider the correlation in the projective space of dimension $2 n-1$ given by

$$
(x, y) \rightarrow(x, y)\left(\begin{array}{cc}
0 & I \\
-I & 0
\end{array}\right)^{t}=(-y, x)^{t},
$$

i.e. $(x, y)$ is mapped on to the hyperplane orthogonal to the column vector at the right. If a point $(p, q)$ lies in the images of the points $\left(x, x T_{i}\right)$ of a component, then $(p, q)$ is orthogonal to $\left(-x T_{i}, x\right)^{t}$ for all $x$.

$$
(p, q)\left(\begin{array}{c}
-T_{i}^{t} x^{t} \\
x^{t}
\end{array}\right)=0 \text { for all } x,
$$

i.e. $p T_{i}^{t} x^{t}=q x^{t}$ for all $x$, i.e. $p T_{i}^{t}=q$, i.e. the image of the component corresponding to the matrix $T_{i}$ consists of points of the form $\left(p, p T_{i}^{t}\right)$ with respect to the same basis of $V_{2 n}$. Thus the components of the dual spread with respect to the same basis in $V_{2 n}$ are given by the transposed matrices $T_{i}^{t}$

If $\pi$ is the translation plane associated with a matrix representative set $\left\{T_{i}\right\}$ as in Result 2, we call the plane associated with the representative set $\left\{T_{i}^{t}\right\}$ the transpose of $\pi$. We now proceed to consider the relation between the collineations of any translation plane and those of its transpose.

Proposition 3. The collineation group fixing the origin of any translation plane is isomorphic to that of its transpose.

Proof. Any collineation of a translation plane fixing the origin is given by a semilinear transformation, say

$$
(x, y) \rightarrow\left(x^{a}, y^{\alpha}\right)\left(\begin{array}{ll}
A & B \\
C & D
\end{array}\right)
$$

where $\alpha$ denotes a field automorphism of the underlying field $F$ of the vector space $V_{n}$ and $A, B, C, D$ are $n \times n$ matrices over the field $F$ [10, p. 52].

Now any component, with points of the form $\left(x, x T_{i}\right)$, is mapped by the given collineation onto a component containing the points of the form

$$
\begin{aligned}
\left(x^{\alpha}, x^{\alpha} T_{i}\right)\left(\begin{array}{ll}
A & B \\
C & D
\end{array}\right) & =\left(x^{a}\left(A+T_{i}^{a} C\right), x^{\alpha}\left(B+T_{i}^{a} D\right)\right) \\
& =\left(x, x\left(A+T_{i}^{a} C\right)^{-1}\left(B+T_{i}^{\alpha} D\right)\right) .
\end{aligned}
$$

But (1) is a collineation of the given plane. Therefore $\left(A+T_{i}^{\alpha} C\right)^{-1}\left(B+T_{i}^{\alpha} D\right)$ is 
again a member of the given representative set if the image component is not the $y$ axis. Otherwise, $\left(A+T_{i}^{\alpha} C\right)$ is singular.

In the transposed translation plane, consider the semilinear transformation given by

$$
(x, y) \rightarrow\left(x^{a}, y^{a}\right)\left(\begin{array}{cc}
0 & I \\
-I & 0
\end{array}\right)\left(\begin{array}{cc}
A & B \\
C & I
\end{array}\right)^{-t}\left(\begin{array}{cc}
0 & -I \\
I & 0
\end{array}\right)
$$

where ${ }^{-t}$ denotes the inverse of the transposed matrix.

Let the point $\left(x, x T_{i}^{t}\right)$ of the component corresponding to the matrix $T_{i}^{t}$ of the transposed translation plane be mapped onto a point, say $\left(\hat{x}, \hat{x} K_{\hat{x}}\right)$. Then

$$
\begin{aligned}
\left(\hat{x}, \hat{x} K_{\hat{x}}\right) & =\left(x^{a}, x^{a} T_{i}^{a t}\right)\left(\begin{array}{cc}
0 & I \\
-I & 0
\end{array}\right)\left(\begin{array}{cc}
A & B \\
C & D
\end{array}\right)^{-t}\left(\begin{array}{cc}
0 & -I \\
I & 0
\end{array}\right) \\
& \Rightarrow\left(-\hat{x} K_{\hat{x}}, \hat{x}\right)\left(\begin{array}{cc}
A^{t} & C^{t} \\
B^{t} & I^{t}
\end{array}\right)=\left(-x^{\alpha} T_{i}^{a t}, x^{a}\right) \\
& \Rightarrow\left(-\hat{x} K_{\hat{x}} A^{t}+\hat{x} B^{t},-\hat{x} K_{\hat{x}} C^{t}+\hat{x} D^{t}\right)=\left(-x^{a} T_{i}^{a t}, x^{a}\right) \\
& \Rightarrow-x^{a} T_{i}^{a t}=-\hat{x} K_{\hat{x}} A^{t}+\hat{x} B^{t} \text { and } x^{a}=-\hat{x} K_{\hat{x}} C^{t}+\hat{x} D^{t} \\
& \Rightarrow\left(\hat{x} K_{\hat{x}} C^{t}-\hat{x} D^{t}\right) T_{i}^{a t}=-\hat{x} K_{\hat{x}} A^{t}+\hat{x} B^{t} \\
& \Rightarrow \hat{x} K_{\hat{x}}\left(A^{t}+\left(t^{t} T_{i}^{a^{t}}\right)=\hat{x}\left(B^{t}+D^{t} T_{i}^{a^{t}}\right)\right. \\
& \Rightarrow \hat{x} K_{\hat{x}}=\hat{x}\left(\left(A+T_{i}^{a} C\right)-1\left(B+T_{i}^{a} D\right)\right)^{t} .
\end{aligned}
$$

Hence

$$
\left(x, x T_{i}^{t}\right) \rightarrow\left(\hat{x}, \hat{x}\left(\left(A+T_{i}^{a} C\right)^{-1}\left(B+T_{i}^{a} D\right)\right)^{t}\right) .
$$

Since $\left(\left(A+T_{i}^{a} C\right)^{-1}\left(B+T_{i}^{a} D\right)\right)^{t}$ is not dependent on $x$ and is also the transpose of a matrix of the representative set as observed from the choice of the given collineation, we have that each point of a component corresponding to the matrix $T_{i}^{t}$ is mapped onto a point of a fixed component. Since $T_{i}^{t}$ is arbitrary, the given mapping preserves the components of the transposed translation plane and, thus, acts as a collineation.

Now the mapping from the collineation group fixing the origin of a translation plane to that of its transpose given by

$$
\left(\begin{array}{ll}
A & B \\
C & D
\end{array}\right) \rightarrow\left(\begin{array}{cc}
0 & I \\
-I & 0
\end{array}\right)\left(\begin{array}{ll}
A & B \\
C & D
\end{array}\right)^{-t}\left(\begin{array}{cc}
0 & -I \\
I & 0
\end{array}\right)
$$

is an isomorphism.

Corollary 1. The isomorphism restricted to the collineation subgroups 
fixing the origin and the $x$-axis takes the form

$$
\left(\begin{array}{ll}
A & 0 \\
C & D
\end{array}\right) \rightarrow\left(\begin{array}{cc}
D^{-t} & 0 \\
\left(A C^{-1} D\right)^{-t} & A^{-t}
\end{array}\right)
$$

and the isomorphism restricted to the collineation subgroups fixing the origin and the $y$-axis takes the form

$$
\left(\begin{array}{ll}
A & B \\
0 & D
\end{array}\right) \rightarrow\left(\begin{array}{cc}
D^{-t} & \left(D B^{-1} A\right)^{-t} \\
0 & A^{-t}
\end{array}\right) .
$$

Although the translation complement and some special subgroups of a translation plane and those of the transposed translation plane are respeco tively isomorphic, there need not be any simple connection between the ways in which isomorphic subgroups act. Thus $\left(\begin{array}{ll}I & 0 \\ C & I\end{array}\right)$ and $\left(\begin{array}{ll}I & 0 \\ 0 & D\end{array}\right)$ both fix the $y$ axis pointwise and, thus, generate a group which does so. However, in the transposed plane, the image of one of them fixes the $y$-axis pointwise and the other fixes the $x$-axis pointwise.

Proposition 4. The right (middle) nucleus of the quasifields of a given translation plane is isomorphic to the middle (right) nucleus of the corresponding quasifields of the transposed plane, having the same unit point $(e, e)$.

Proof. The right nucleus of the quasifield of the given translation plane corresponds to the group of $((\infty),[0,0])$ homologies which are of the form $\left(\begin{array}{ll}I & 0 \\ 0 & D\end{array}\right)$. Now by the isomorphism of Proposition 3, this collineation subgroup is mapped onto the collineation subgroup of the form

$$
\left(\begin{array}{cc}
D^{-t} & 0 \\
0 & I
\end{array}\right)
$$

in the transposed translation plane, which is precisely the $((0),[0])$ homologies in this plane. Hence, the right nucleus of the translation plane corresponds to the middle nucleus of the corresponding quasifield in the transposed translation plane. Similarly follows the other result.

Proposition 5. The transpose of the following classes of planes belong to the same class (1) Desarguesian planes, (2) nearfield planes, (3) semifield planes, (4) generalised Hall planes, (5) Luneburg planes, (6) Bol planes, (7) generalised André planes, and (8) C-planes.

Proof. First three follow from [3, Lemma 11] and the fact that $T_{i} \rightarrow$ $T_{i}^{t}$ preserves addition and, anti-isomorphically, matrix multiplication. The fourth, fifth, sixth and seventh follow from Proposition 3 together with [7, Theorem 1], [9, Theorem 12.3], [5, Theorem 2.1], and [11, Theorem 5.3], respectively. Finally the eight follows from straightforward computations 
of the transposed inverses of the respective sets of the translation $C$ planes $[10$, p. 80].

Acknowledgements. The author wishes to thank his supervisor, Prof. M. Venkataraman for his helpful guidance, and Prof. R. P. Burn and Dr. T. V. S. Jaganathan for valuable suggestions. Special thanks are due to the referee for his substantial improvement of this paper.

\section{REFERENCES}

1. J. André, Über nicht-Desarguessche Ebenen mit transitiver translations. gruppe, Math. Z. 60 (1954), 156-186. MR 16, 64.

2. R. H. Bruck and R. C. Bose, The construction of translation planes from projective spaces, J。Algebra 1 (1964), 85-102. MR 28 \#4414.

3. - Linear representations of projective planes in projective spaces, J. Algebra 4 (1966), 117-172. MR 33 \#4777.

4. A. Bruen, Spreads and a conjecture of Bruck and Bose, J. Algebra 23 (1972), 519-537. MR $46 \# 8035$.

5. R. P. Burn, Bol quasi-fields and Pappus' theorem, Math. Z. 105 (1968), 351-364. MR 37 \#5778.

6. P. Dembowski, Finite geometries, Ergebnisse der Mathematik und ihrer Grenzgebiete, Band 44, Springer-Verlag, Berlin and New York, 1968. MR 38 \#1597.

7. P. B. Kirkpatrick, Generalization of Hall planes of odd order, Bull。Austral。 Math. Soc. 4 (1971), 205-209. MR 43 \#10348.

8. D. E. Knuth, Finite semifields and projective planes, J. Algebra 2 (1965), 182-217. MR 31 \#218.

9. H. Lüneburg, Die Suzukigruppen und ihrer Geometrien, Vorlesung Sommersemester 1965 in Mainz, Lecture Notes in Math., vol. 10, Springer-Verlag, Berlin and New York, 1965. MR $34 \# 7634$.

10. M. L. Narayana Rao, D. J. Rodabaugh, F. W. Wilke and J. L. Zemmer, A new class of finite translation planes obtained from the exceptional near-fields, J. Combinatorial Theory Ser. A 11 (1971), 72-92. MR 43 \#5408.

11. T. G. Ostrom, Finite translation planes, Lecture Notes in Math., vol. 158, Springer-Verlag, Berlin and New York, 1970. MR 43 \#1031.

DEPARTMENT OF MATHEMATICS, MADURAI UNIVERSITY, MADURAI-21, INDIA

Current address: Department of Mathematics, University of Illinois at Chicago Circle, Chicago, Illinois 60680 